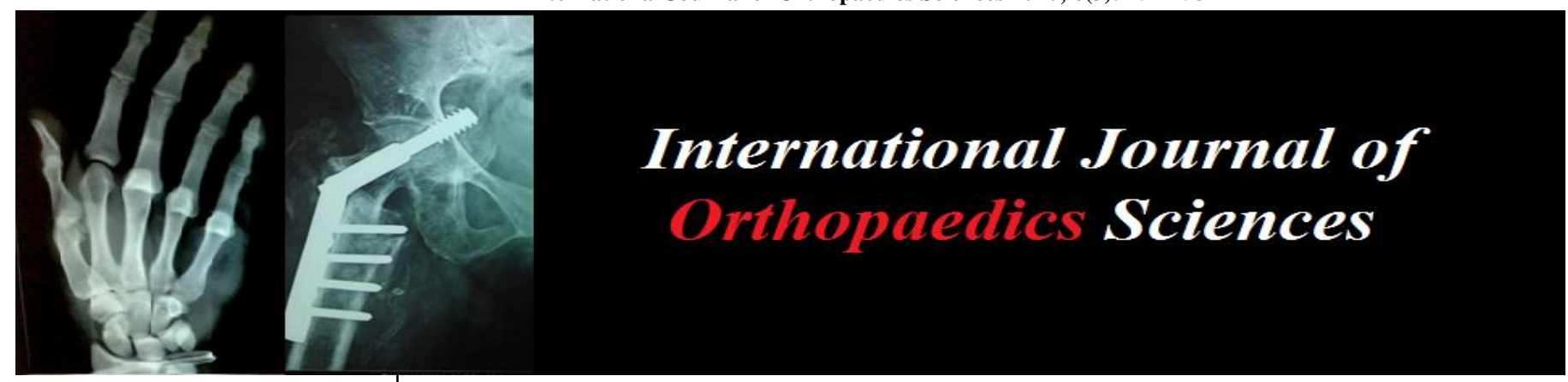

E-ISSN: 2395-1958

P-ISSN: 2706-6630

IJOS 2020; 6(3): 754-758

(C) $2020 \mathrm{IJOS}$

www.orthopaper.com

Received: 05-05-2020

Accepted: 12-06-2020

Dr. Shashi Vardhan Mudavath Senior Resident, Department of Orthopaedics, Esic Medical College and Hospital,

Sanathnagar, Hyderabad,

Telangana, India

Dr. Ranjit Kumar Yalamanchili Assistant Professor, Department of Orthopaedics, Esic Medical College and Hospital,

Sanathnagar, Hyderabad,

Telangana, India

Dr. Vutkuru Sri Ravindranath Professor, Department of Orthopaedics, Esic Medical College and Hospital,

Sanathnagar, Hyderabad,

Telangana, India

Dr. Venu Madhav Vuthpala Department of Orthopaedics, Esic Medical College and Hospital, Sanathnagar, Hyderabad, Telangana, India

Corresponding Author: Dr. Ranjith Kumar Yalamanchili Assistant Professor, Department of Orthopaedics, Esic Medical College and Hospital Sanathnagar, Hyderabad, Telangana, India

\section{Covid-19 preparedness for Orthopaedic practice in public health care system}

\author{
Dr. Shashi Vardhan Mudavath, Dr. Vutkuru Sri Ravindranath, Dr. \\ Ranjith Kumar Yalamanchili and Dr. Venu Madhav Vuthpala
}

DOI: https://doi.org/10.22271/ortho.2020.v6.i31.2279

\section{Abstract}

The impact of COVID - 19 has a challenge to balance the work of combating the virus on one hand and continuing to treat the routine non-covid problems on the other hand. Apart from trauma and other emergencies which require immediate surgery, there are a lot of acute and chronic painful conditions along with post-operative follow up cases which cannot be postponed from orthopaedic consultation for a long time. Universal guidelines for COVID-19 preparedness stands good for both private and public health care systems, but addressing social distancing, personal hygiene and health awareness among patients attending public hospitals pose a great challenge. Due to the huge patient load in public hospitals, compared to private hospitals, these government run institutions are particularly at risk of becoming a hotspot for virus transmission if adequate precautions are not taken. In this article, we discuss feasible cost-effective strategies against COVID transmission to continue treating orthopedic patients in a safe environment.

Keywords: COVID-19, Orthopaedics, public healthcare

\section{Introduction}

The impact of COVID-19 on the world has been huge, affecting many developing and developed nations. The healthcare sector now has a challenge to balance the work of combating the virus on one hand and continuing to treat the routine non-covid problems on the other hand. The emergence of Corona virus pandemic (COVID-19) has impacted orthopaedic surgery all over. Following the recommendations of the Government of India and guidelines laid down by major Orthopaedic associations, all major private and public hospitals have started to delay or postpone elective surgeries during the COVID-19 outbreak. As the pandemic weans, there will be a surge in Orthopaedic patients requiring admissions for surgeries, sometimes with significantly increased morbidity. As per record, for a population of over 125 crores, there are approximately 20,000 Orthopaedic surgeons, i.e. one surgeon for every 62,500 people ${ }^{[1]}$. The Orthopaedic outpatient and emergency departments both in public and private hospitals are often flooded with patients, requiring emergency and non-emergency care. Apart from trauma and other emergencies which require immediate surgery, there are a lot of acute and chronic painful conditions along with post-operative follow up cases which cannot be postponed from Orthopaedic consultation for a long time. Hence, continuation of out-patient services during covid-19 pandemic and lockdown is essential.

The hospitals have been recognized as a potential source of contracting the virus. Many healthcare professionals across the globe are reported to have contracted the disease at workplace and thus there is an equal risk for the patients and their attendants as well, if continued with regular practice methods. India has mixed health care system of private and public health care service providers, of which the latter cater to majority population. Universal guidelines for COVID-19 preparedness stands good for both private and public health care systems, but addressing social distancing, personal hygiene and health awareness among patients attending public hospitals pose a great challenge. Bearing in mind about the risks involved, the essential patient care both surgical and non-surgical need to be provided while conserving resources and creating a safe environment for patients and the health care workers. 
Although the literature on precautionary measures to be followed during the pandemic are on the rise, following these guidelines is a bigger challenge, particularly in public health sector. Due to the huge patient load in public hospitals, compared to private hospitals, these government run institutions are particularly at risk of becoming a hotspot for virus transmission if adequate precautions are not taken. At our hospital which is a tertiary public health care center, we have adopted feasible cost-effective strategies against COVID transmission to continue treating orthopedic patients in a safe environment.

\section{Strategies during out-patient care}

In view of huge number patient visits daily, the hospital should have a separate screening for all people entering the gate at the entrance itself and a separate dedicated block for treatment of COVID patients away from the main building. All the personnel entering the hospital premises should be educated and encouraged to take self-assessment on participatory disease surveillance tool - "Arogya-Setu" mobile application on their mobile phones ${ }^{[2]}$. Patients should be instructed to visit the designated COVID clinic if they have any symptoms of fever, cough, or shortness of breath. Hand wash facility with soap at the entrance of hospital and each OPD, should be made available which not only enforces the patients to wash their hands, but also be a part of health education to teach importance of hand hygiene. Necessary measures by marking designated boxes so as to maintain social distancing between the patients in queue and also between the patient and the doctor during consultation is arranged. [Fig.1\&2] The clinician should use protective gears like N-95 masks, latex gloves, face shields or goggles which can be worn over the spectacles while in Out-Patient department. The doctor can make use of personal scrubs which can be washed in campus and can change clothes before leaving the hospital. All the healthcare staff are advised to avoid wearing accessories like wrist watches and wedding rings. Mobile phones can be wrapped securely with a transparent plastic sheet while use in the hospital and can be discarded before going home [Fig 3]. Only one attendant is allowed with the patient at any time. 'No Touch Technique' is employed with minimalistic handling of patient files and $\mathrm{x}$ rays. We can ask either the patient or attendant to handle the documents and hold the $\mathrm{x}$-ray/CT/MRI against the view box, if needed. Any kind of paper transfer from patient to doctor should be minimized to curtail transmission of fomites. It is advisable to look for the clinical signs that can be elicited without touching the patient and check Range of movements of affected joint by demonstrating them. It is the time where in one should rely on clinical diagnosis and proceed with treatment rather than use investigations to look for evidence of the disease, wherever possible. This should be followed to minimize the exposure to laboratory technicians or the radiology staff and other healthcare personnel. As surgery and hospital stay increases the risk of transmission, conservative management can be adopted wherever feasible. Patients can be given video links on 'you-tube' to follow physiotherapy at home. Steroid injections can be used in certain cases to provide prolonged symptomatic relief until definitive procedure is performed. Upper limb injuries should be managed with work up and pre-anaesthetic evaluation to admit just before day of surgery to minimize hospital stay. Proper and thorough counselling of patients with chronic ailments and those who need elective surgeries should be done to avoid repeated visits to the hospital.

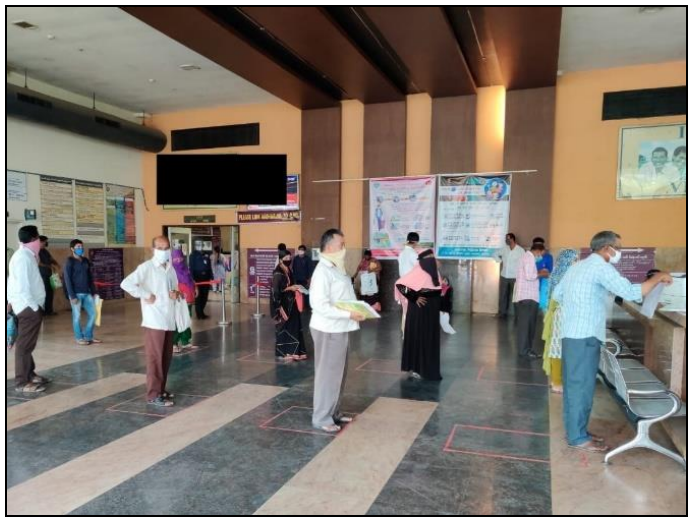

Fig 1: Designated boxes for patients to stand in queue maintaining adequate Social distance in the Registration area of Out-patient department.

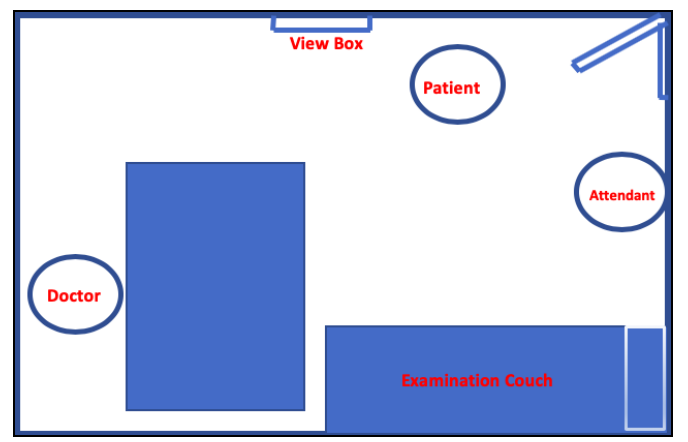

Fig 2: Social distancing inside doctor chamber in OPD

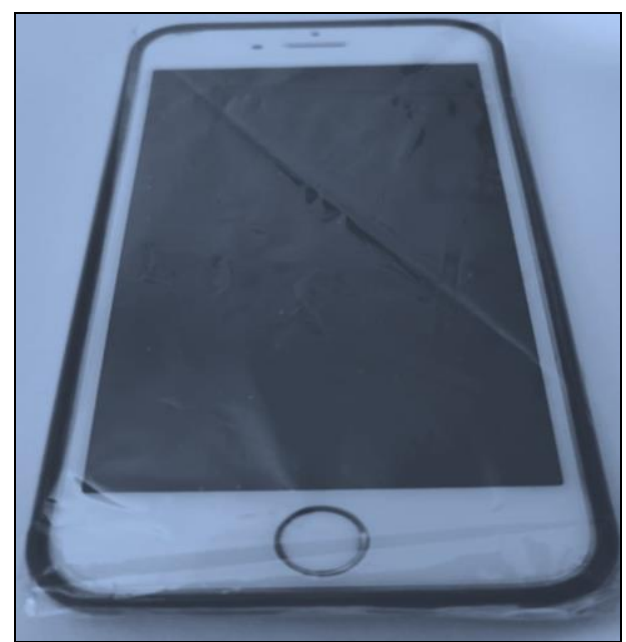

Fig 3: Using disposable transparent plastic wrap around cell phones.

\section{Strategies during in-patient services \\ Bedside Management}

Minimize the admission to hospital unless absolutely necessary. Prior to admission, adequate history is to be obtained regarding symptoms, history of travel, any contact with a suspected patient, area of residence like containment areas or red zones and work area. In case of any positive history or if symptomatic, both patient and attendant should be moved to an isolation ward and treated as a positive case until the test results are available. It is advisable to educate patients to have only one attendant throughout the hospital stay and to obtain similar history from the attendant. The attendant is also refrained from leaving the hospital premises to avoid fresh contact. All patients and attendants are provided with masks and are instructed to use it during all times. They are counselled and educated about the hand hygiene aspects and risk of COVID-19 transmission if they do 
not follow the precautions strictly. As there will be minimal or no elective cases, hospital census might allow to adjust beds in the ward with adequate distancing. Cardiac tables can be used to barricade the Nursing station desk, allowing social distance to health care workers from patients and their attendants. [Fig 4] Case sheets and other paper-work at patient side are to be handled by using latex gloves as these can act as fomites carrying virus. All the surfaces like door handles, taps, etc., which patients come in contact are regularly disinfected. All the patients in ward should be daily assessed with respect to COVID-19 symptoms. It is seen that the admitted patients receive early intervention and discharge, so as to decrease the hospital stay.

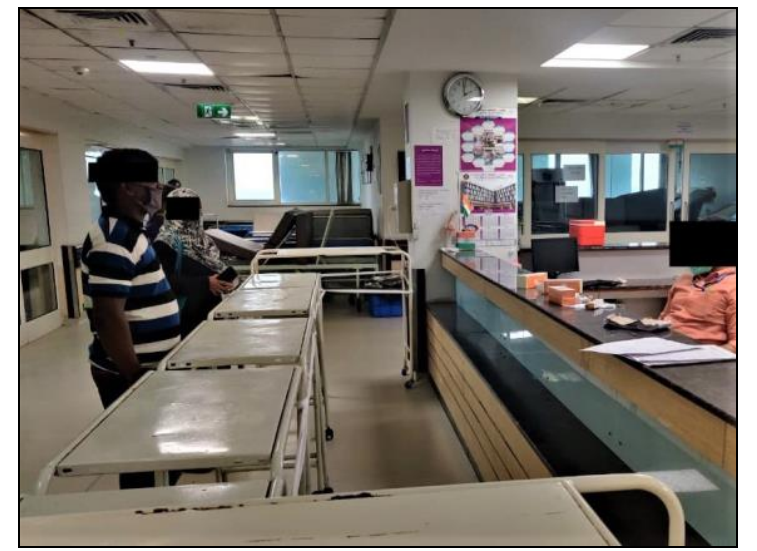

Fig 4: Use of cardiac tables as barricades near nursing stations

\section{Pre-operative evaluation}

Apart from routine investigations, thorough pre-anaesthetic check-up is mandated taking into account the age and other comorbidities. The patient should undergo protocol-based testing for corona virus disease. Wherever possible for asymptomatic patients from containment zone or history of positive contact, COVID-19 antigen and antibody testing SARS-CoV real time reverse transcription polymerase chain reaction (RT-PCR) test ${ }^{[3]}$ should be done to evaluate active contagion or exposure to the virus. The rapid kit tests to check the antibodies are not recommended for use in the surgical patients due to their variable results ${ }^{[4]}$. Widespread testing by RT-PCR for all the patients is a bigger task for the government and sometimes may not be possible in case of an emergency where one cannot wait for the test results. In such cases, proper history about symptoms, contacts, travel and place of residence might be helpful. Chest CT, a routine imaging tool, is a relatively easy alternative for patients with suspicion or emergency in public health care settings. In a recent study on over 1000 COVID infected patients from Wuhan, researchers concluded that CT Chest could be used as the primary screening tool for COVID-19 as it has high sensitivity ${ }^{[5]}$.

Basing on availability of the resources, it is advisable to carry out CT scan of the chest whenever articular CT scan is ordered for surgical planning or in cases of significant history or COVID-19 RT-PCR test if possible. This would minimize the aerosol spread in OT, where the chances of transmission are high.

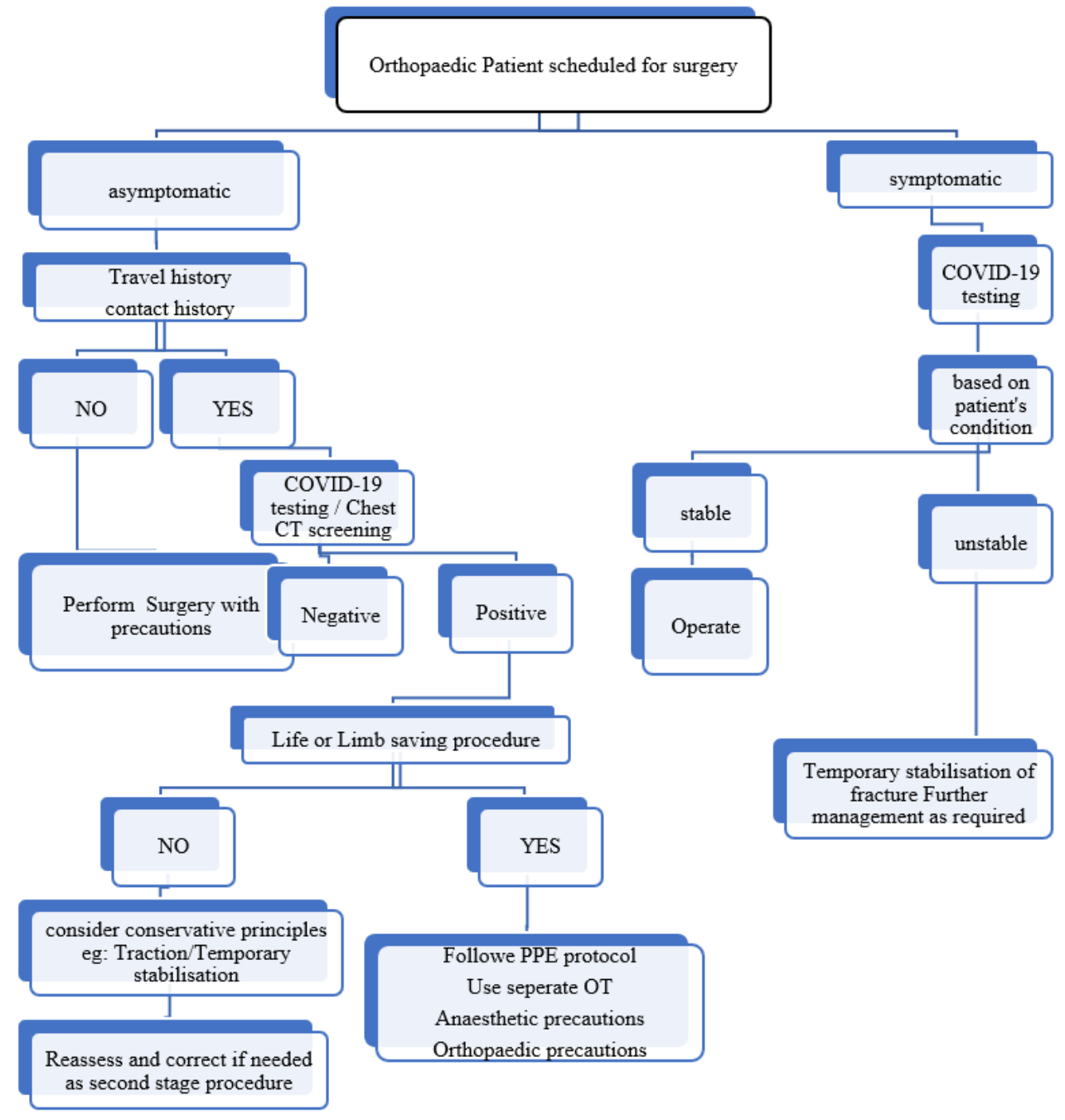

Flow chart 1: COVID preparedness in Orthopedic practice 


\section{Inside the Operation theatre}

\section{Theatre settings}

A dedicated OT set up with Laminar flow system, negative pressure environment and frequent air exchange are recommended. Based on resources, operative theatre with separate entry and exit should be dedicated for the surgery involving suspected or infected patients of COVID-19. Rodrigues-Pinto et al. ${ }^{[6]}$ have given a detailed account of the surgical team flow for the orthopaedic surgery in a COVID19 dedicated operating theatre and divided the complex into five zones: i) Entry dressing room, ii) Anteroom, iii) Operating Room, iv) Exit room, v) shower room and v) Exit. These protocols can be adapted according to the infrastructure available.

The number of patients listed for surgery in a day should be kept to minimum and only half of the available theatres be used for that day. Operation theatres should be used on alternate days and the used theatre should be sealed and decontaminated thoroughly giving a gap of at least 24 hours between procedures. The operating personnel are to be provided with adequate protective gears like N95 masks, goggles or face shields, full PPE kit in case of suspected patient or who has not been tested in view of emergency surgery. All theatre staff should be trained in proper Donning and Doffing techniques and made sure these are followed strictly. Proper disposal of contaminated materials like mops/ gauze pieces and surgical gowns should be employed. After the surgery, the theatre should be decontaminated/ fumigated thoroughly.

\section{Anesthetic concerns}

The Anaesthesiologists and the theatre staff are at risk during intubation and airway management for General anaesthesia ${ }^{[7]}$. Hence, regional anaesthesia like nerve blocks for upper limbs, spinal/epidural for lower limbs is preferred wherever possible. The patients inside the OR are provided with face masks/face shields. In cases where General anaesthesia is unavoidable, it is carried out in theatre with minimal number of staff by using an aerosol limiting chamber over patients face which can decrease the droplet spread ${ }^{[8]}$ [Fig 5]. Exit and entry of any person for the next $20 \mathrm{~min}$ after intubation should be restricted; staff members are not allowed to go in or come out of the theatre ${ }^{[9]}$. The same applies when the patient is extubated. It is mandatory that necessary checks be placed to ensure that equipment such as anaesthesia machines used to intubate or ventilate patients are thoroughly decontaminated as per anaesthesia society and CDC guidelines ${ }^{[9]}$.

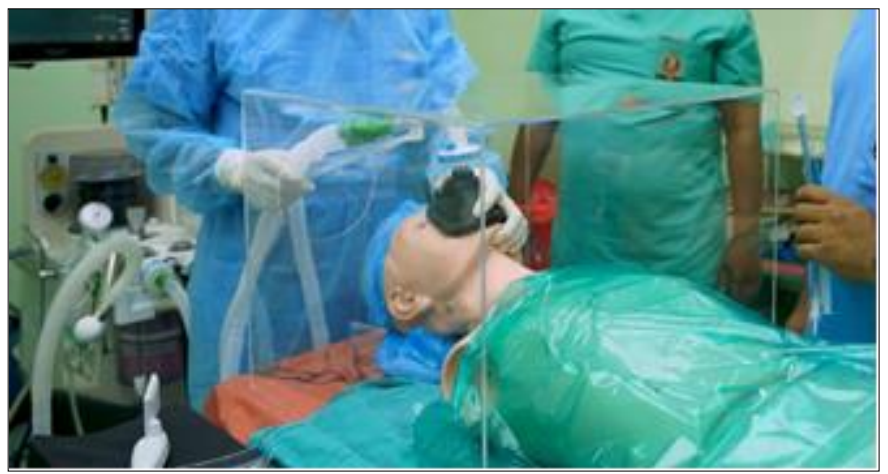

Fig 5: Usage of aerosol limiting chamber during airway management by anesthetists.

\section{Orthopaedics procedures}

Operating techniques are modified to reduce the contamination and thus the risk. Wherever possible, percutaneous methods of reduction and fixation are preferred like closed reduction and $\mathrm{k}$-wire fixation of distal radius, MIPPO technique for distal femur or proximal tibia. These methods are less invasive and thus decrease the exposure as the skin and soft tissues act as a barrier. The usage of diathermy has been reported to increase the transmission and so should be limited and should only be used along with suction if deemed necessary. Power drills and reamers are better avoided as these are aerosol generating procedures rendering the theatre staff at risk. Use of osteotomes, nibblers instead of oscillating saw as in the past should be encouraged. Similarly, a Gigli saw can be used in amputations. Wherever possible the exposed bone should be covered with wet mop to prevent blood spillage. Pulse lavage should be replaced with saline in a syringe for irrigation ${ }^{[10]}$.

\section{Post-operative period}

After the surgery, patient should be shifted to the ward from post-operative ward as early as possible based on patient's condition and comorbidities. Hospital stay should be minimized by discharging the patient on $2^{\text {nd }}$ post-operative day after wound examination. It is advisable to minimize the use of intravenous drugs based on local policies. The use of technology like tele-medicine and video consultations can help in following up patients in order to avoid revisits to the hospital ${ }^{[11]}$. One stop visits to the hospital can be arranged for the postoperative patients by creating a separate follow up clinic in the outpatient department. Patients should be evaluated for common complications and instructed clearly about the next visits only if necessary.

One of the most important aspect in orthopedic post-operative patients is physiotherapy and rehabilitation which must not be neglected. The patient can be shared with video links or materials with description of certain exercises and rehabilitation protocols which in collaboration with telemedicine can be a better replacement of follow up visits ${ }^{[12]}$.

These patients should be followed up frequently and a timely audit of the medical and surgical outcome can help us make future plans and modifications about the management protocols during this COVID-19 pandemic. In view of a possible second wave of cases similar to the past pandemics that the world has experienced and the difficulty in developing and vaccinating the whole world, it is advisable to adhere to such precautionary measures for a couple of months or years to come ${ }^{[13]}$.

\section{Conclusion}

The pandemic of COVID-19 has given the healthcare fraternity an unexpected challenge to face. These are difficult times where one should be vigilant about the changes going on all over the world in terms of morbidity, mortality associated with the disease and different ways to tackle the situation. There is an overwhelming need for scientific evidence and literature on this issue but as we are in an early phase of pandemic with short time for research, the need cannot be met. At this juncture, it is imperative that we share knowledge and experience with fellow colleagues across the globe so that it can help each other in creating a safe working environment until the disease is eradicated. Here, we have discussed our practical experiences and difficulties faced in public health care system during the COVID-19 pandemic and strategies that can be adapted to continue orthopedic services in a safe and effective manner without compromising on patient care. 


\section{Acknowledgement}

The guidelines provided here are not truly evidence based. These are based on individual opinion and experience at our institution, besides some information available from guidelines by expert bodies like IOA (Indian Orthopaedic Assosciation), CDC, WHO, Ministry of Health \& Family welfare - Government of India.

\section{Recommendations}

- Hand washing must be encouraged for everyone before entering the hospital premises.

- Queue management system should be practiced strictly in the Out-patient department.

- Tele-medicine should be utilized wherever possible to counsel, educate and advising patients.

\section{References}

1. Jain AK. Current state of Orthopaedic education in India. Indian J Orthop. 2016; C50:341-344.

2. Garg S, Bhatnagar N, Gangadharan N. A Case for Participatory Disease Surveillance of the COVID-19 Pandemic in India. JMIR Public Health Surveill. 2020; 6(2):e18795. doi:10.2196/18795.

3. Centers for disease control and prevention. Novel Coronavirus (2019-nCoV) Situation Summary; 2019. https://www.cdc.gov/coronavirus/2019ncov/casesupdates/summary

4. Mallapaty S. Will antibody tests for the coronavirus really change everything? Nature, 2020. https://doi.org/10.1038/d41586-020-01115-Z

5. Ai T, Yang Z, Hou $\mathrm{H}$ et al. Correlation of Chest CT and RT-PCR Testing in Coronavirus Disease 2019 (COVID19) in China: A Report of 1014 Cases [published online ahead of print, 2020 Feb 26]. Radiology. 2020;200642. doi:10.1148/radiol.2020200642

6. Rodrigues-Pinto R, Sousa R, Oliveira A. Preparing to perform trauma and orthopaedic surgery on patients with COVID-19. J Bone Joint Surg Am, 2020. https://doi.org/10.2106/JBJS.20.00454.

7. Tran $\mathrm{K}$ et al. Aerosol generating procedures and risk of transmission of acute respiratory infections to healthcare workers: a systematic review. PloS One. 2012; 7(4):e35797.

https://doi.org/10.1371/journal.pone.0035797. Published online 2012 April 26 PMCID: PMC3338532.

8. Malik JS, Jenner C, Ward PA. Maximising application of the aerosol box in protecting healthcare workers during the COVID-19 pandemic [published online ahead of print, 2020 Apr 29]. Anaesthesia, 2020. 10.1111/anae.15109. doi:10.1111/anae.15109

9. COVID-19: infection prevention and control (IPC). Public health England. 10 January 2020. Last updated 12 April, 2020

10. Raghavan R, Middleton PR, Mehdi A. Minimising aerosol generation during orthopaedic surgical procedures- Current practice to protect theatre staff during Covid-19 pandemic. J Clin Orthop Trauma. 2020; 11(3):506- 507. doi:10.1016/j.jcot.2020.04.024

11. Iyengar K, El-Nahas W. A brief guide to telephone medical consultation. $\mathrm{Br} \mathrm{J}$ Healthc Manag. 2020; https://doi.org/10.12968/bjhc.2020.0032.

12. Iyengar KP, Nadkarni JB, Ivanovic $\mathrm{N}$, Mahale A. Targeted early rehabilitation at home after total hip and knee joint replacement: does it work? Disabil Rehabil. 2007; https://doi.org/10.1080/09638280600841471

13. Vannabouathong $\mathrm{C}$, Devji $\mathrm{T}$, Ekhtiari S et al. Novel coronavirus COVID-19: current evidence and evolving strategies. J Bone Joint Surg Am. 2020. https://doi.org/10.21 06/JBJS.20.00396. 